\title{
Distribution of the molecular absorption in front of the quasar B0218+357
}

\author{
S. Muller ${ }^{1}$, M. Guélin ${ }^{2}$, F. Combes ${ }^{3}$, and T. Wiklind ${ }^{4}$ \\ 1 Academia Sinica Institute of Astronomy and Astrophysics (ASIAA), PO Box 23-141, Taipei, 106 Taiwan \\ e-mail:muller@asiaa.sinica.edu.tw \\ 2 Institut de Radio Astronomie Millimétrique (IRAM), 300 rue de la piscine, 38406 St Martin d'Hères, France \\ 3 Observatoire de Paris, LERMA, 61 Av. de l'Observatoire, 75014 Paris, France \\ ${ }^{4}$ ESA Space Telescope Division, STScI, 3700 San Martin Drive, Baltimore, MD 21218, USA
}

Received 16 February 2007 / Accepted 3 April 2007

\section{ABSTRACT}

\begin{abstract}
The line of sight to the quasar B0218+357, one of the most studied lensed systems, intercepts a $z=0.68$ spiral galaxy, which splits its image into two main components $\mathrm{A}$ and $\mathrm{B}$, separated by ca. $0.3^{\prime \prime}$, and gives rise to molecular absorption. Although the main absorption component has been shown to arise in front of image $\mathrm{A}$, it is not established whether some absorption from other velocity components is also occuring in front of image $\mathrm{B}$. To tackle this question, we have observed the $\mathrm{HCO}^{+}(2-1)$ absorption line during the commissioning phase of the new very extended configuration of the Plateau de Bure Interferometer, in order to trace the position of the absorption as a function of frequency. Visibility fitting of the self-calibrated data allowed us to achieve position accuracy between $\sim 12$ and 80 mas per velocity component. Our results clearly demonstrate that all the different velocity components of the $\mathrm{HCO}^{+}(2-1)$ absorption arise in front of the south-west image $\mathrm{A}$ of the quasar. We estimate a flux ratio $f_{\mathrm{A}} / f_{\mathrm{B}}=4.2_{-1.0}^{+1.8}$ at $106 \mathrm{GHz}$.
\end{abstract}

Key words. galaxies: quasars: individual: B0218+357 - galaxies: quasars: absorption lines - techniques: interferometric

\section{Introduction}

Molecular absorption at intermediate redshifts has been detected only in a few objects (e.g. Wiklind \& Combes 1995, 1996a, 1997, 1998; Kanekar et al. 2005). Among these, three absorption systems are caused by a galaxy lying on the line-of-sight of a quasar and acting as a gravitational lens. Two systems, one in front of PKS1830-211 (at $z=0.89$ ), the other in front of $\mathrm{B} 0218+357$ (at $z=0.68$ ) are detected at millimeter wavelengths in the lines of several molecules, including $\mathrm{HCO}^{+}, \mathrm{HCN}$ and $\mathrm{H}_{2} \mathrm{CO}$.

The light rays associated with the quasar PKS1830-211 form two bright images that probe different regions of the intervening galaxy. This provides information on the latter's kinematics, its mass, as well as on the physical and chemical conditions in its interstellar medium (Wiklind \& Combes 1998; Muller et al. 2006). These two papers showed that the two $z \sim 0.89$ absorption components of PKS1830-211 are associated each with one of the two gravitational images.

The second mm-absorption system occurs in the line of sight to B0218+357 and is caused by a galaxy at a redshift of $z=0.68466$ (Browne et al. 1993; Carilli et al. 1993; Wiklind $\&$ Combes 1995). Two bright images of the quasar, hereafter referred to as A (to the $\mathrm{SW}$ ) and $\mathrm{B}$ (to the $\mathrm{NE}$ ), have been resolved at radio $\mathrm{cm}$ wavelengths (O'Dea et al. 1992; Patnaik et al. 1993). The distance AB between the two images is $\sim 0.3^{\prime \prime}$, the smallest angular separation among the known galaxy-mass lenses. The flux ratio is $f_{\mathrm{A}} / f_{\mathrm{B}} \sim 3$ between $5 \mathrm{GHz}$ and $22 \mathrm{GHz}$.
Image B lies in the center of an Einstein ring whose diameter is similar to the distance AB. Each image reveals intricate substructures at very high angular resolution (Patnaik et al. 1995; Biggs et al. 2003). The constraints provided by the complex image pattern and by the time variability of the background source flux, make of B0218+357 one of the best objects to measure $H_{0}$ at intermediate redshifts (Biggs et al. 2003; Wucknitz et al. 2004; York et al. 2005). Deep ACS/HST observations of B0218+357 by York et al. (2005) reveal that the lensing object is a spiral galaxy seen nearly face-on and whose center lies close to image $\mathrm{B}$, the center of the Einstein ring. According to the lensing model proposed by Wucknitz et al. (2004), image A is located at about $2 \mathrm{kpc}$ from the center of the lensing mass distribution, and image B at $0.4 \mathrm{kpc}$. HI absorption is detected over a velocity width of about $100 \mathrm{~km} \mathrm{~s}^{-1}$ (Carilli et al. 1993; Kanekar et al. 2003), in front of image A (Carilli et al. 2000), although some absorption may also occurs in front of the Einstein ring (Kanekar et al. 2003). $\mathrm{H}_{2} \mathrm{CO}\left(2_{12}-2_{11}\right)$ absorption has been observed by Menten \& Reid (1996) towards image A, with a total width of $\sim 12 \mathrm{~km} \mathrm{~s}^{-1}$, much narrower than the HI profile. Because of limited signal-to-noise ratio, they could however not exclude the possibility of absorption in front of B.

Figure 1 shows the $\mathrm{HCO}^{+}(1-2)$ absorption profile towards B0218+357 observed with a high sensitivity and velocity resolution (Muller et al., in prep.). Like the HCN (1-2) profile, it shows at least four velocity components over a width of $25 \mathrm{~km} \mathrm{~s}^{-1}$, much broader than that of $\mathrm{H}_{2} \mathrm{CO}\left(2_{12}-2_{11}\right)$. The component with the deepest absorption matches in width and velocity the $\mathrm{H}_{2} \mathrm{CO}$ 


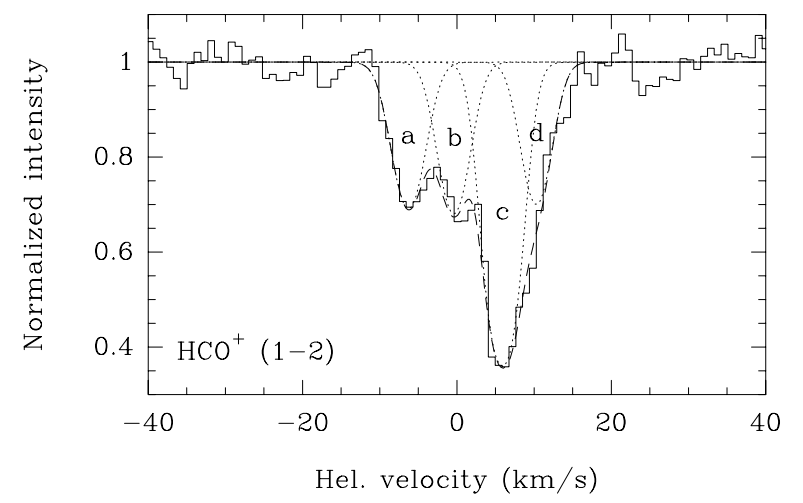

Fig. 1. Spectrum of the $\mathrm{HCO}^{+}(1-2)$ absorption towards $\mathrm{B} 0218+357$ as observed with the PdBI in a compact configuration. Gaussian fits of the different velocity components $\mathrm{a}, \mathrm{b}, \mathrm{c}, \mathrm{d}$ are overlaid as dashed lines.

$\left(2_{12}-2_{11}\right)$ absorption profile and most probably originates like this latter, in front of image A. The other three components, however, had not been observed so far with a high angular resolution and their location, in front of A, B, or of the Einstein ring remained unknown. Yet, the knowledge of this location is essential for the interpretation of the molecular absorption data in terms of line opacities, molecular column densities and gas kinematics.

In this letter, we present observations of the $\mathrm{HCO}^{+}(1-2)$ line toward $\mathrm{B} 0218+357$, obtained with the new, extended configuration of the Plateau de Bure Interferometer, which allow us to trace the position of all four absorption components.

\section{Observations and data analysis}

In the frame of a survey of molecular absorption lines towards $\mathrm{B} 0218+357$, we have observed the $\mathrm{HCO}^{+}(1-2)$ line in June 2005 and July 2006, with a compact configuration of the IRAM Plateau de Bure Interferometer (PdBI). These observations were self-calibrated on the continuum source, which was not resolved with the $\sim 4^{\prime \prime}$ FWHP synthetized beam. The resulting average $\mathrm{HCO}^{+}(1-2)$ spectrum is shown in Fig. 1. The absorption extends over $\sim 25 \mathrm{~km} \mathrm{~s}^{-1}$ and can be decomposed into four Gaussian velocity components, with $F W H M$ of $4-5 \mathrm{~km} \mathrm{~s}^{-1}$.

We have further observed on November 8th 2005 the $\mathrm{HCO}^{+}(1-2)$ line with an extended configuration (maximum projected baseline $B_{\max }$ of $385 \mathrm{~m}$ ) and on November 9th 2005 with the new, very extended configuration of the PdBI $\left(B_{\max }=\right.$ $650 \mathrm{~m})$. We emphasize that these observations were the first science data acquired with the new extended configuration and that they were performed during its commissioning phase. In particular, the instrument baselines were not yet properly calibrated, the on-source integration time was limited (110 min in each configuration), and the weather not at its best (rms phase deviation prior to self-calibration of $\sim 50^{\circ}$ on the longest baselines). Therefore, the data needed to be self-calibrated on the continuum source, which was half-resolved in the EW and NEW directions.

As in the June and July sessions, we observed simultaneously a $540 \mathrm{MHz}$-wide frequency band centered at $105.690 \mathrm{GHz}$, consisting of two overlapping $320 \mathrm{MHz}$-wide continuum sub-bands (L03 and L04) with a channel spacing of $2.5 \mathrm{MHz}\left(7.1 \mathrm{~km} \mathrm{~s}^{-1}\right)$, and a $80 \mathrm{MHz}$-wide sub-band (L01) centered on the redshifted $\mathrm{HCO}^{+}(1-2)$ line frequency $v=$ $105.882 \mathrm{GHz}$, with a channel spacing of $0.31 \mathrm{MHz}\left(0.9 \mathrm{~km} \mathrm{~s}^{-1}\right)$. The high frequency edge of the continuum band was dropped and its bandwidth restricted to $440 \mathrm{MHz}$ in order to avoid contamination by the $\mathrm{HCO}^{+}(1-2)$ line.

The method used to self-calibrate on the half-resolved continuum source was similar to the one used by Muller et al. (2006), for localizing the absorption components in front of PKS1830-211. The data from November 8th and 9th were calibrated separately, using the GILDAS/CLIC software, with the following procedure: in a first step, the radio frequency (RF) bandpass of L03 and L04 was calibrated directly on the B0218+357 continuum signal. The main purpose of using these absorption-free bands was to correct for short term phase and intensity variations linked to atmosphere fluctuations by selfcalibrating on the continuum. L01 was calibrated in RF separately, using low order polynomials (first degree in amplitude and third degree in phase). The narrowness of the absorption line $(<10 \mathrm{MHz})$ with respect to the L01 bandwidth $(80 \mathrm{MHz})$ ensured that the calibration was not badly affected by the absorption features. Note that we choose a baseline-based RF calibration, rather than an antenna-based, as the signal intensities for the longest baselines were severely affected by atmospheric phase decorrelation.

Next, the continuum sub-bands were self-calibrated in amplitude and phase by calculating the complex gains corresponding to a system of two point-like sources separated by $\triangle \mathrm{RA}=$ 309 mas, $\Delta \mathrm{Dec}=128$ mas, and with a flux ratio of 4.2 (a justification of theses values will be given in Sect. 3, and/or can be found in the reviews by Wucknitz et al. 2004; Mittal et al. 2006a,b). The total continuum intensity ( $\sim 0.4 \mathrm{Jy})$ was normalized to unity. The gains calculated for the continuum subbands were then applied to the visibilities of the L01 sub-band channels. Finally, the continuum visibilities, as derived from the double-source model, were subtracted from the calibrated L01 visibilities.

The calibrated L01 datasets from November 8th and 9th were then combined. The visibilities corresponding to uv radii larger than $200 \mathrm{~m}$ were fitted, channel by channel, with a single pointlike source representing the position and strength of the absorption signal. Visibilities with shorter uv radii were discarded, as they bring little information on the signal position. The fitted source intensities showed a small offset $(\sim 10 \%$ of the total continuum) with respect to zero (the continuum level as defined from the continuum sub-bands) - probably the result of residual IF and/or RF bandpass calibration errors. This was taken care of by averaging the visibilities of the L01 channels that are free of any absorption and by subtracting the so-computed residual continuum visibility from all L01 channels. The fit of the point-like absorption sources was then repeated.

Figure 2 shows the result of the final fits. The upper plot (i) shows the absorption profile as a function of velocity, while the two middle plots ( $i i$ and $i i i)$ show the position of the absorption (or, more exactly, the position of the centroid of the absorption) as a function of velocity. The removal of the continuum insures that zero intensity $(0)$ and zero offset $(0,0)$ effectively correspond to the intensity and position of the centroid of the continuum sources. The source position accuracy in the individual velocity channels was calculated by the GILDAS fitting routine UVFIT. It can be crudely expressed as $\sim \theta_{\text {beam }} / 2$ SNR, where $\theta_{\text {beam }}$ is the FWHP of the synthesized beam $\left(\simeq 1.2 \times 0.7^{\prime \prime}\right.$, PA $176^{\circ}$ ) and SNR, the signal-to-noise ratio on the source intensity. The noise level, per $0.9 \mathrm{~km} \mathrm{~s}^{-1}$ velocity channel, is $3.4 \%$ of the continuum intensity. For all channels with absorption signal $\left(-10<V_{\mathrm{HEL}}<+12 \mathrm{~km} \mathrm{~s}^{-1}\right)$, the fitted phases indicates that, within the uncertainties, the absorption arises from a small size region that coincides in position with image $\mathrm{A}$. We further 


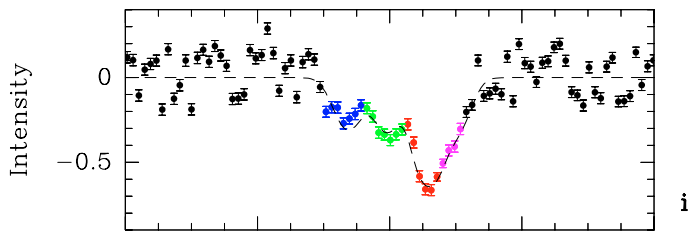

i)

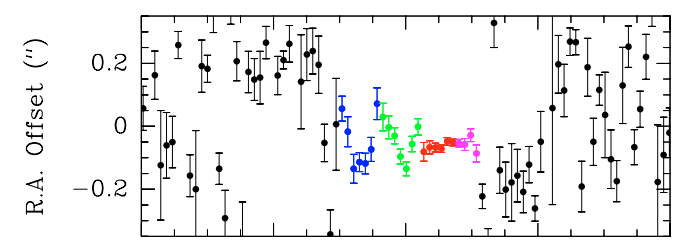

ii)
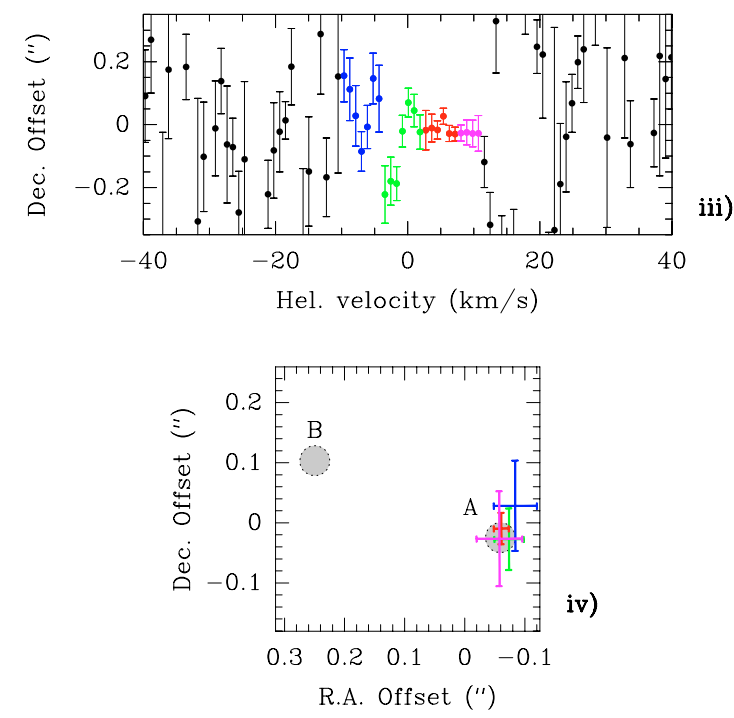

Fig. 2. Relative intensity and position of the centroid of the $\mathrm{HCO}^{+}(1-$ 2) absorption as derived from the fit of a point like-source to the selfcalibrated visibilities, for each velocity channel. i) The intensity scale is zero at the continuum level and at -1 for full absorption. The profile of the absorption, as fitted in Fig. 1, is overlaid as a dashed line. ii, iii) Position offsets relative to the centroid of the continuum. iv) Position of the four Gaussian velocity components, compared with the locations of the two lensed images of the quasar (grey disks, not to scale).

averaged the position offsets per velocity components, with weights equal to the square of the intensity of the Gaussian profiles. The average positions are given in Table 1 and Fig. 2iv.

\section{Discussion}

Our data show that all the molecular absorption components (from $V_{\mathrm{HEL}}=-10$ to $+12 \mathrm{~km} \mathrm{~s}^{-1}$ ) originate in front of image $\mathrm{A}$. This is not surprising since image $\mathrm{A}$ appears more obscured in the visible than image B (Grundahl \& Hjorth 1995). As was mentioned, Menten \& Reid (1996) have already shown that the $V_{\mathrm{HEL}} \sim 5 \mathrm{~km} \mathrm{~s}^{-1} \mathrm{H}_{2} \mathrm{CO}$ absorption component arose in front of image A.

The background radio source presents a core-jet morphology that is reflected in both lensed images in the form of substructures. Those consist mainly in two main components, labelled A1 and A2 (and B1 and B2), which are separated by $\sim 1.4$ mas (Patnaik et al. 1995). High sensitivity radio imaging reveals in addition the presence of a weak, relatively extended (10-15 mas) knotty jet and of a possible counterjet (Biggs et al. 2003). Our source positioning accuracy is not good enough to spatially resolve any of these sub-components. However, the jet has a steeper spectrum than the core and its emission, which is already weak in the radio domain should be negligible at millimeter wavelengths. The relevant size of the background continuum, regarding millimeter absorption, should be $<4$ mas $^{2}$. Assuming a flat universe, with standard cosmological parameters $\left(H_{0}=70 \mathrm{~km} \mathrm{~s}^{-1} \mathrm{Mpc}^{-1}, \Omega_{\mathrm{M}}=0.3, \Omega_{\Lambda}=0.7\right)$, the angular size scale is $7.1 \mathrm{kpc} /{ }^{\prime \prime}$ at a redshift $z=0.68$. The mm continuum emission of image A should therefore have an extent lower than $\sim 30 \mathrm{pc}^{2}$. This value, corrected from the filling factor, gives an estimate of the size of the molecular absorbing clouds.

It is difficult to determine a direct value of the flux ratio $f_{\mathrm{A}} / f_{\mathrm{B}}$ from our current data. Nevertheless, we have repeated the self-calibration procedure described in Sect. 2, by varying the flux ratio in the source model. The fitted positions of the absorption, averaged over $V_{\mathrm{HEL}}=-10$ to $+12 \mathrm{~km} \mathrm{~s}^{-1}$, were then compared to the position of image A set by the source model (see Fig. 3). Given the size of the continuum source, the average position of the absorption is consistent with the position of image $\mathrm{A}$ for $f_{\mathrm{A}} / f_{\mathrm{B}}=4.2_{-1.0}^{+1.8}$. We emphasize at this point that the average position derived in this way changes by less than \pm 20 mas when $f_{\mathrm{A}} / f_{\mathrm{B}}$ varies from 3 to 6 , i.e. by the range of possible $f_{\mathrm{A}} / f_{\mathrm{B}}$ ratios, so that our conclusion that all the absorption arises in front of A is robust. Similarly, the self-calibration method that we have used depends little on the continuum source model, in particular on the distance between A and B, so that the uncertainties on this distance do not affect our results.

Although indirect, our measurement of the flux ratio $f_{\mathrm{A}} / f_{\mathrm{B}}$ is the first at frequencies higher than $22 \mathrm{GHz}$. The value of 4.2 is slightly higher than those measured at 15 and $22 \mathrm{GHz}$ with the VLA (O'Dea et al. 1992; Patnaik et al. 1993; Biggs et al. 1999), and almost twice higher than those observed around $2 \mathrm{GHz}$ (Mittal et al. 2006a). This is consistent with the model developed by Mittal et al. (2006b), where image A is obscured at radio frequencies by a HII region associated with the molecular cloud. We note that the $f_{\mathrm{A}} / f_{\mathrm{B}}$ ratio might be affected by the time variability of the quasar, due to the time difference $\Delta t$ between the transit times of the light in A and B. The time delay, however, is relatively short ( $\Delta t \sim 10$ days, Biggs et al. 1999), making the chance of a factor of 1.5-2 variation of the quasar intensity in less than $\Delta t$ rather unlikely.

Adopting $f_{\mathrm{A}} / f_{\mathrm{B}}=4.2$ and assuming a filling factor $f_{\mathrm{c}}=1$, we derive a maximum optical depth $\tau_{\max }=1.5$ for component $\mathrm{c}$, i.e. the peak opacity is large. Conversely, a lower limit to the filling factor $f_{\mathrm{c}}$ can be derived by assuming $\tau_{\max } \rightarrow \infty$; this yields $f_{\mathrm{c}} \geq 0.77$. The $\mathrm{H}_{2} \mathrm{CO}$ (Menten \& Reid 1996), $\mathrm{NH}_{3}$ (Henkel et al. 2005) and $\mathrm{H}_{2} \mathrm{O}$ (Combes \& Wiklind 1997) absorptions are probably caused by the same cloud as component c. For the other velocity components, the filling factors and/or the peak opacities must be lower. A filling factor $f_{\mathrm{c}} \simeq 0.9$ is consistent with the fact that image $\mathrm{A}$, despite a high column density of absorbing gas, is strongly attenuated on optical $V$-band images, with respect to $H$-band images, but still visible. It might also naturally explain why the optical distance AB ( 317 mas, York et al. 2005) appears lower than in radio ( $\sim 334$ mas). The optical barycenter of image A should indeed be shifted closer to image B, if the absorption occurs mostly on the opposite border.

By co-adding all our observations in the compact configuration of the PdBI at the frequency of the $\mathrm{HCO}^{+}(1-2)$ line, we do not detect any new absorption feature, outside the $V_{\mathrm{HEL}}=-10$ to $+12 \mathrm{~km} \mathrm{~s}^{-1}$ components just described, over the range $V_{\mathrm{HEL}}=$ -200 to $+1300 \mathrm{~km} \mathrm{~s}^{-1}$, and this down to a level of $3 \sigma=3.7 \%$ of the continuum at a velocity resolution of $7.1 \mathrm{~km} \mathrm{~s}^{-1}$. As the intervening galaxy is seen face-on, it is unlikely that the difference in velocity between positions $\mathrm{A}$ and $\mathrm{B}$, located at either sides of the 
Table 1. Average positions of the different absorption components. For comparison, the positions of images A and B, as fixed in our continuum source model, are indicated. Offsets are given relatively to the barycenter of the continuum emission.

\begin{tabular}{cccccc}
\hline \hline $\begin{array}{c}\text { Velocity } \\
\text { component }\end{array}$ & $\begin{array}{c}V_{\mathrm{HEL}^{\dagger}} \\
\left(\mathrm{km} \mathrm{s}^{-1}\right)\end{array}$ & $\begin{array}{c}\text { Absorption } \\
\text { depth }^{\dagger}\end{array}$ & $\begin{array}{c}\Delta V_{1 / 2}^{\dagger} \\
\left(\mathrm{km} \mathrm{s}^{-1}\right)\end{array}$ & $\begin{array}{c}\text { RA offset } \\
(\mathrm{mas})\end{array}$ & $\begin{array}{c}\text { Dec offset } \\
\text { (mas) }\end{array}$ \\
\hline $\mathrm{a}$ & -6.3 & 0.31 & 4.7 & $-84 \pm 36$ & $+28 \pm 75$ \\
$\mathrm{~b}$ & -0.3 & 0.32 & 4.5 & $-73 \pm 24$ & $-28 \pm 51$ \\
$\mathrm{c}$ & +5.9 & 0.63 & 4.5 & $-60 \pm 12$ & $-11 \pm 26$ \\
$\mathrm{~d}$ & +10.3 & 0.30 & 4.4 & $-57 \pm 38$ & $-27 \pm 79$ \\
$\mathrm{a}+\mathrm{b}+\mathrm{c}+\mathrm{d}$ & -10 to +12 & & & $-61 \pm 15$ & $-15 \pm 32$ \\
\hline Image A & & & & -59 & -25 \\
Image B & & & & +250 & +103 \\
\hline
\end{tabular}

$\dagger$ From the fit of the spectrum obtained in the compact configuration (cf. Fig. 1).

\#ith respect to the total continuum intensity.

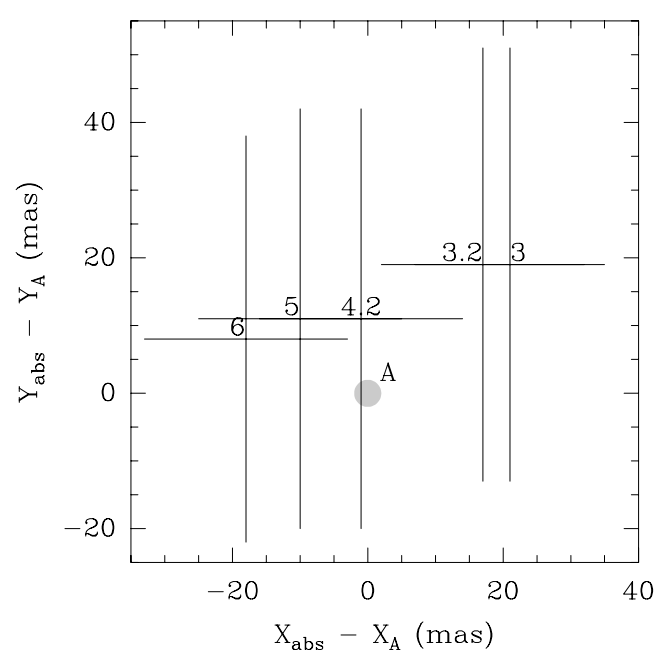

Fig. 3. Average positions of the $\mathrm{HCO}^{+}(1-2)$ absorption features obtained when varying the flux ratio $f_{\mathrm{A}} / f_{\mathrm{B}}$ (indicated above each position) in the source model used for the self-calibration.

center of the galaxy, is more than $200 \mathrm{~km} \mathrm{~s}^{-1}$. $\mathrm{HCO}^{+}$is known to be easily detectable in absorption in the Galaxy, even in diffuse molecular clouds (Lucas \& Liszt 1996). Therefore, either the region intercepted by the light path associated with image B and located at $\sim 400 \mathrm{pc}$ from the center of the galaxy, is free or almost free of molecular gas $\left(\tau_{\mathrm{HCO}+}<0.2\right)$, or the filling factor is low $\left(f_{\mathrm{c}}<0.2\right)$.

\section{Conclusion}

Sensitive observations of the quasar $\mathrm{B} 0218+357$ in the $\mathrm{HCO}^{+}(1-2)$ line, redshifted by $z=0.68$, show at least four velocity components in absorption with velocities between $V_{\mathrm{HEL}}$ $=-6$ to $+10 \mathrm{~km} \mathrm{~s}^{-1}$ and widths $\simeq 5 \mathrm{~km} \mathrm{~s}^{-1}$. By fitting the visibilities obtained from new observations with the very extended configuration of the Plateau de Bure Interferometer, we show unequivocally that all these components arise in front of the SW gravitational image (A) of the quasar. We see no other $\mathrm{HCO}^{+}$absorption components over a range $V_{\mathrm{HEL}}=-200$ to $+1300 \mathrm{~km} \mathrm{~s}^{-1}$, that could arise from image $\mathrm{A}$, from the $\mathrm{NE}$ image $\mathrm{B}$, or from the weak Einstein ring visible at radio frequencies. This implies either a low average column density of
$\mathrm{HCO}^{+}(\tau<0.2)$ in front of $\mathrm{B}$ and, presumably, a low average molecular hydrogen column density, or a low filling factor $\left(f_{\mathrm{c}}<\right.$ 0.2 ). We derive a flux ratio $f_{\mathrm{A}} / f_{\mathrm{B}}=4.2_{-1.0}^{+1.8}$ for the two main components, $\mathrm{A}$ and $\mathrm{B}$, at $106 \mathrm{GHz}$, which is slightly higher than those derived between 15 and $22 \mathrm{GHz}$ and almost twice larger than observed at frequencies around $2 \mathrm{GHz}$. This measurement, strictly speaking, applies only to Nov. 8 and 9, 2005, the dates of our extended configuration observations.

Acknowledgements. The results presented here are based on observations carried out with the IRAM Plateau de Bure Interferometer. We would like to thank all the people who have been working on the extension of the interferometer tracks and, more particularly, the IRAM staff that has carried out these observations. We thank the referee, Prof. Karl Menten, for constructive comments. IRAM is supported by INSU/CNRS (France), MPG (Germany) and IGN (Spain).

\section{References}

Biggs, A. D., Browne, I. W. A., Helbig, P., et al. 1999, MNRAS, 304, 349 Biggs, A. D., Wucknitz, O., Porcas, R. W., et al. 2003, MNRAS, 338, 599 Browne, I. W. A., Patnaik, A. R., Walsh, D., \& Wilkinson, P. N., et al. 1993, MNRAS, 263, L32

Carilli, C. L., Rupen, M. P., \& Yanny, B. 1993, ApJ, 412, L59

Carilli, C. L., Menten, K. M., Stocke, J. T., et al. 2000, Phys. Rev. Lett., 85, 5511 Combes, F., \& Wiklind, T. 1997, ApJ, 486 L79

Grundahl, F., \& Hjorth, J. 1995, MNRAS, 275, 67

Henkel, C., Jethava, N., Kraus, A., et al. 2005, A\&A, 440, 893

Kanekar, N., Chengalur, J. N., de Bruyn, A. G., \& Narasimha, D., et al. 2003, MNRAS, 345, L7

Kanekar, N., Carilli, C. L., Langston, G. I., et al. 2005, Phys. Rev. Lett. PhRv, 95, L1301

Lucas, R., \& Liszt, H. 1996, A\&A, 307, 237

Menten, K. M., \& Reid, M. J. 1996, ApJ, 465, L99

Mittal, R., Porcas, R., Wucknitz, O., Biggs, A., \& Browne, I., et al. 2006a, A\&A, 447, 515

Mittal, R., Porcas, R., \& Wucknitz, O. 2006b, A\&A, 465, 405

Muller, S., Guélin, M., Dumke, M., Lucas, R., \& Combes, F., et al. 2006, A\&A, 458,417

O’Dea, C. P., Baum, S. A., Stanghellini, C., et al. 1992, AJ, 104, 1320

Patnaik, A. R., Browne, I. W. A., King, L. J., et al. 1993, MNRAS, 261, 435

Patnaik, A. R., Porcas, R. W., \& Browne, I. W. A., et al. 1995, MNRAS, 274, L5

Wiklind, T., \& Combes, F. 1995, A\&A, 299, 382

Wiklind, T., \& Combes, F. 1996a, A\&A, 315, 86

Wiklind, T., \& Combes, F. 1996b, Nature, 379, 139

Wiklind, T., \& Combes, F. 1997, A\&A, 328, 48

Wiklind, T., \& Combes, F. 1998, ApJ, 500, 129

Wucknitz, O., Biggs, A. D., \& Browne, I. W. A., et al. 2004, MNRAS, 349, 14

York, T., Jackson, N., Browne, I. W. A., Wucknitz, O., \& Skelton, J. E. 2005, MNRAS, 357, 124 